\title{
Health promotion and disease prevention in general practice and primary care: a scoping study
}

\author{
Stephen Peckham ${ }^{1,2}$, Alison Hann ${ }^{3}$, Sally Kendall ${ }^{4}$ and Steve Gillam ${ }^{5,6}$ \\ ${ }^{1}$ Professor of Health Policy, Centre for Health Services Studies, University of Kent, Canterbury, UK \\ ${ }^{2}$ Professor of Health Policy, Department of Health Services Research and Policy, London School of Hygiene and \\ Tropical Medicine, London, UK \\ ${ }^{3}$ Senior Lecturer, Public Health, Policy and Social Sciences, Swansea University, Swansea, UK \\ ${ }^{4}$ Professor of Community Nursing and Public Health, Centre for Health Services Studies, University of Kent, Canterbury UK \\ ${ }^{5}$ General Practitioner, Luton, UK \\ ${ }^{6}$ Department of Public Health and Primary Care, University of Cambridge, Cambridge, UK
}

\begin{abstract}
This paper reports the findings of a scoping review on the organisation and delivery of health improvement activities in general practice and the primary healthcare team. The project was designed to examine who delivers these interventions, where they are located, what approaches are developed in practices and how individual practices and the primary healthcare team organise such public health activities and how these contribute to health improvement. Our focus was on health promotion and prevention activities and aimed to identify the current extent of knowledge about the health improvement activities in general practice and the wider primary healthcare team. Many of the research studies reviewed had some details about the type, process, location or who provided the intervention. Little attention is paid in the literature to examining the impact of the organisational context on the way services are delivered or how this affects the effectiveness of health improvement interventions in general practice. We found that the focus of attention is mainly on individual prevention approaches with practices engaging in both primary and secondary prevention. Although many GPs do not take a population approach and focus on individual patients some do see health promotion as an integral part of practice - whether as individual approaches to primary or secondary health improvement or as a practice-based approach to improving the health of their patients. Based on our analysis we conclude that there is insufficient good evidence to support many of the health improvement interventions undertaken in general practice and primary care.
\end{abstract}

Key words: General practice; health improvement; primary care

Received 27 July 2016; revised 18 January 2017; accepted 10 July 2017; first published online 11 August 2017

\section{Introduction}

Internationally there has been growing interest in the public health role of primary care and how best to achieve a stronger orientation to prevention and health promotion services for patients (World Health Organization, 1978; 2005). The increasing

Correspondence to: Professor Stephen Peckham, Centre for Health Services Studies, George Allen Wing, University of Kent, Canterbury, CT2 7NF, UK. Email: s.peckham@kent.ac.uk focus on chronic health management models including prevention and self-management, reductions in demand through self-care and prevention have become important issues for policy and practice in many countries (Brady, 2013). In the United Kingdom, prevention is a key element of new models of practice across primary and secondary care but with a particular emphasis on developing the public health role of general practice (NHS England, 2014). As managers of care it has been hypothesised that GPs paid under a capitation 
system would be more likely to focus on prevention than if they were paid on a fee-for-service basis, but there is little evidence to support this view (Peckham and Gousia, 2014). In the United Kingdom the government also introduced a pay for performance system - the Quality and Outcomes Framework (QOF), which was designed to improve practices performance in a number of areas of preventive care, reduce variations in care and improve quality of care. However, the impact on health outcomes has been fairly limited and the extent to which QOF has improved the quality of primary care, particularly in relation to prevention, is highly debatable (Peckham and Wallace, 2010; Dixon et al., 2011; Langdown and Peckham, 2013).

Since the mid-1990s professional bodies have argued that prevention should be a constituent element of normal professional practice of GPs and nurses and that prevention and health promotion should be an integral part of general practice (Royal College of General Practitioners, 2007; Royal College of Nursing, 2007; Watson, 2008; Peckham et al., 2011). In the United Kingdom, successive governments have emphasised the important role of GPs in promoting their patients' health and in 2010 the newly elected Coalition Government specifically promoted greater GP involvement in public health (HM Government, 2010). In the White Paper Healthy Lives, Healthy People (Sec of State $2010 \mathrm{~cm} 7985$ ) and other public statements, the government outlined an increased role for GPs and general practices in public health. In addition, since January 2012 there has been a call for all NHS professionals to 'Make every contact count' making prevention a part of every patient contact with the NHS including opportunistic advice as well as part of planned support sessions for patients [Department of Health, 2012; National Institute for Health and Clinical Excellence (NICE), 2013].

However, concerns have been raised about whether it is appropriate for increasing elements of health promotion, and public health more generally to be undertaken in general practice, given the limited consultation time available and a growing workforce 'crisis'. Despite the recognition of increasing pressures on general practice, new models of care being developed within the NHS especially for primary and community care involve a strong emphasis on prevention and health promotion (NHSE, 2016). Similarly, a recent Health Education England Primary Care Workforce Commission report highlighted the need for more nurses to undertake health promotion in primary and community care settings and the continuing importance of the prevention role in primary care (Health Education England, 2015). This, of course, is by no means a new idea and harks back to the 80 s and 90 s when practice nurses were called on to manage health checks in primary care to prevent coronary heart disease with GPs financially incentivised to undertake prevention and health promotion prevention and health promotion interventions and screening (Peckham et al., 2011).

Yet there remain key questions about the both the effectiveness of prevention and health promotion activities undertaken by GPs and nurses, their focus and what the level of such activity is in practice (Peckham et al., 2011). In particular, studies suggest that workload issues, lack of time, limited knowledge, and lack of skill remain key reasons for not engaging in prevention interventions in primary care (Brotons et al., 2005; Rubio-Valera et al., 2014). In order to examine some of these issues NIHR HS\&DR funded this scoping project to provide a synthesis of the literature on prevention and health promotion in practice (Peckham et al., 2015). The aim was to identify what is known about the effectiveness of prevention and health promotion activities in general practice and the wider primary healthcare team. The key objectives were to provide an overview of the range and type of prevention and prevention and health promotion activities undertaken, identify gaps in knowledge and areas for further empirical research. While the study had a primarily UK focus the findings from the review provide more general insights to the role of primary care in prevention and public health and the lack of evidence about how these activities should most effectively be organised and delivered.

\section{Methods}

The aim was to conduct a synthesis of the research evidence and wider literature on the delivery and organisation of prevention and health promotion activities in primary care. We focussed on the contribution of activities undertaken in or associated 
with general practice, wider practice and the primary healthcare team but excluding mental health prevention. We undertook a comprehensive search of the literature. Our methodological approach was based on the standard methodology for scoping reviews (Arksey and O'Malley, 2005; Anderson et al., 2008). Reviews of public health prevention and health promotion interventions pose numerous challenges due to multi-component interventions, diverse study populations, multiple outcomes measured, wide range of approaches and study designs used, and the effect of context on intervention design, implementation and effectiveness (Jackson and Waters, 2004). An initial scoping of the literature identified in excess of 20,000 articles that may be relevant to the topic area and we developed strategies designed to manage paper selection and data extraction including limiting the search to UK only articles. The search was run on four databases, namely Medline, Embase, Global Health and CINAHL Plus, using strategies designed specifically for each database combining two searches - one with broad terms 'prevention', 'health improvement' and 'health promotion and one focussing on a number of key areas alcohol and drug addiction, brief interventions, exercise therapy, immunisation, lifestyle, risk reduction, screening and smoking cessation (see Supplementary Material Appendices 1 and 2). Results were limited to academic articles published in the English language between 1990 and 2012. This yielded 16,791 references. Paper selection was conducted in three sequential stages - by title, by abstract and by full text. At each stage, the inclusion criteria are as follows:

- Is it focussed on primary care?

- Is it health improvement (defined as primordial, primary, secondary or tertiary prevention, and also encompassing health promotion)?

- Is it, or could it be, related to service delivery or organisation?

- Does it report research findings, or alternatively, does it contain a description of health improvement activities undertaken in practice?

The 16,791 references were divided so that in the title selection stage, the title of each article was reviewed separately by three reviewers. If any one reviewer considered an article to be relevant, it was progressed to the abstract selection stage. In the abstract stage, references were divided so that each abstract was reviewed separately by two reviewers.
If both reviewers considered an article to be relevant, it was progressed to the full-text stage. In the full-text stage, data from each paper was extracted by individual reviewers. This resulted in a final selection of 658 papers for inclusion in the review, of which 347 were included in the evidence synthesis with the remainder providing contextual and descriptive background information. Full details of our search strategy and methods for paper selection are set out in the published report (Peckham et al., 2015) and details of numbers of papers and the selection process are shown in Figure 1.

\section{Description of papers}

We distinguished between three broad areas of preventive and health promoting activities - screening, primary prevention and secondary prevention. ${ }^{1}$ The use of these three areas emerged during the review process as the most coherent way of segmenting the substantial amount of literature. There were 148 papers concerning screening, although only 84 of these specifically examined research relating to a screening programme. The remainder were predominantly general descriptive accounts or commentaries. We identified 326 papers on primary prevention (164 included in the evidence synthesis) including 50 papers on child health, 49 on smoking, 40 on exercise and weight interventions, and 22 on vaccination. There were 64 papers that we left unclassified in terms of the intervention focus including studies on welfare advice and community initiatives. Finally, we identified 126 papers on secondary prevention, with cardiovascular disease (CVD) being the most common topic but there were also papers specifically focussing on weight and exercise for people with symptomatic disease. As described in the published report, the papers covered a huge range of interventions, types of interventions and locations in which interventions were carried out (Peckham et al., 2015).

\section{Findings}

In this paper we summarise the key findings by prevention and health promotion category. However, it

\footnotetext{
${ }^{1}$ Primary prevention is an activity or action taken before the onset of symptoms, whereas secondary prevention seeks to limit the impact of a disease that has already manifested.
} 


\section{Stephen Peckham et al.}

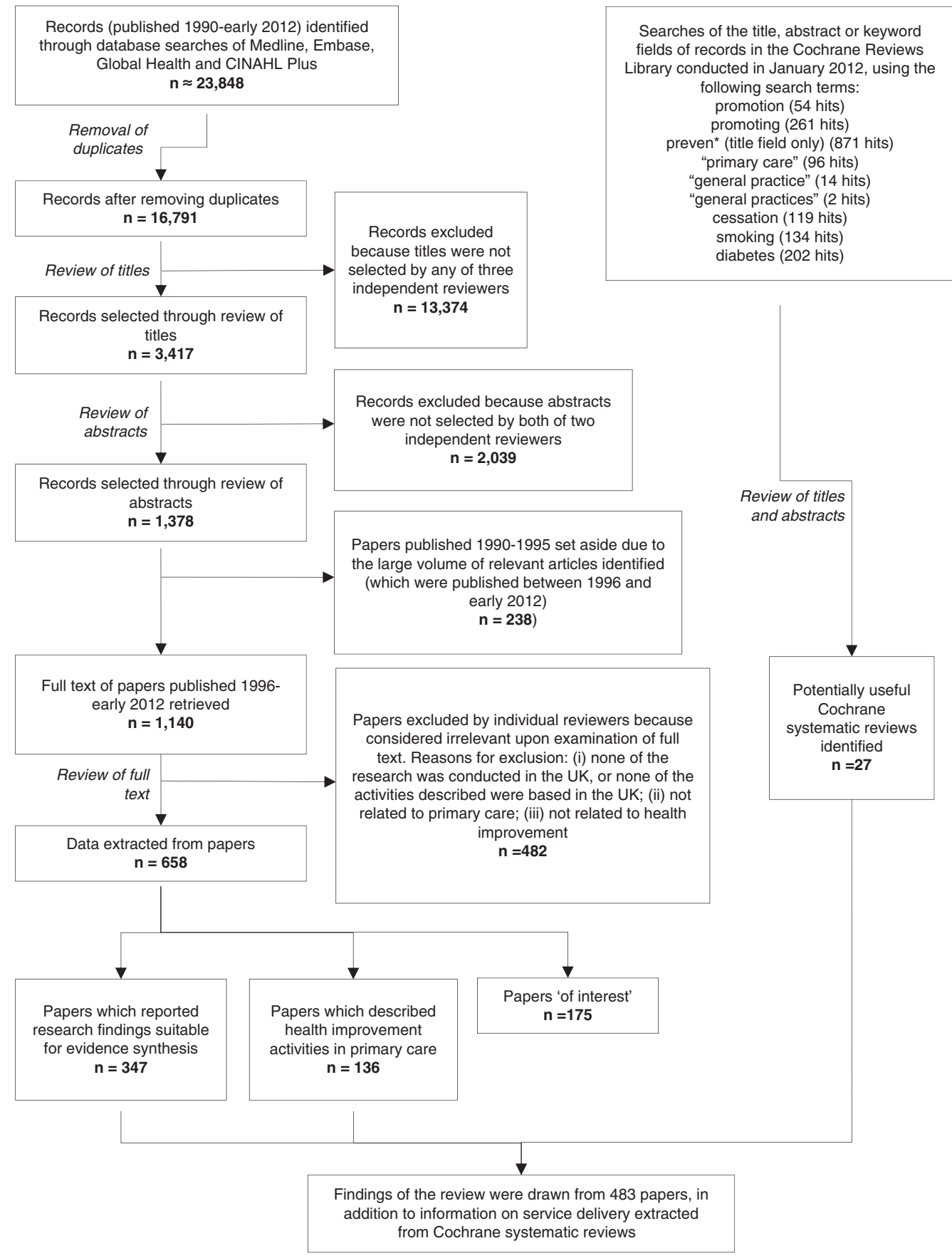

Figure 1 Flow diagram of review process

Primary Health Care Research \& Development 2017; 18: 529-540 
was not always easy to distinguish between primary and secondary prevention in the allocation of papers, as some interventions might appear to be primary in nature, they might have been applied in practice to patients with an existing health problem, for example CVD. In reporting the findings we predominantly draw on the synthesis reported in our substantive report (Peckham et al., 2015) except where it is useful to refer directly to specific studies.

\section{Screening}

We divided the 148 screening papers into systematic screening and opportunistic screening, though the boundary was blurred because opportunistic screening may be used in general practice to increase screening uptake for systematic screening programmes (Health and Social Care Information Centre, 2012). We also categorised them by topic, 84 being evidence papers with the remainder being contextual or descriptive. Of the evidence papers, 16 examined sexual health screening, 13 diabetes screening, eight cervical cancer and six breast cancer with the rest predominantly CVD. NHS Health Checks combined accounted for nine papers. While many screening programmes are managed nationally there are several programmes in which general practice has a role; including cervical cancer screening, NHS Health Checks, child health surveillance, diabetes and CVD screening, and health checks for people aged over 75 years. Most studies focussed on screening coverage and women's experiences, including such aspects as informed consent, reasons for non-attendance rather than aspects of how services were organised and delivered (Peckham et al., 2015).

Screening programmes for CVD, diabetes and kidney disease have become an increasingly important part of UK screening programmes that involve general practice although there is inconsistency in the way screening activities were delivered despite attempts by programme designers to standardise it (Peckham et al., 2015). The initial national health checks programme was for people with learning disabilities as distinct from the later NHS Health Checks Programme discussed below. There was a very wide variation in service delivery of the health checks programme for people with learning disabilities but it had been successful in detecting unmet health needs and provision of appropriate support. The checks were carried out in different locations (eg, GP surgeries and people's homes), and were carried out by a diversity of practitioners including GPs, practice nurses and community nurses (Robertson et al., 2011). The ADDITION study examined screening and intensive management for people with diagnosed diabetes found only a small reduction in cardiovascular events and death, but this was not statistically significant and screening was not associated with a reduction in mortality over a 10-year period (Simmons et al., 2012).

In 2009 the national NHS Health Check programme was introduced in England to undertake screening of people aged between 40 and 74 for risk of heart disease, stroke, diabetes and kidney disease in primary care. There has, though, been substantial controversy over the effectiveness of NHS health checks (Capewell et al., 2015; Waterall et al., 2015). Over time these screening activities have begun to be carried out in a wide variety of locations, including general practice, community pharmacy, workplaces and in one instance a mobile bus and by a variety of healthcare professionals and non-professionals (Peckham et al., 2015). Where the screening took place in general practice, in the majority of cases, it was carried out by the practice nurse, although healthcare assistants and primary care staff were also mentioned. In community locations, there was a very wide range of personnel including practice nurses, health visitors, staff from an independent sector organisation and district nurses working with lay health workers but we did not identify any research that compared different delivery and organisational arrangements. Since completing the review the range of service providers has diversified following the transfer of commissioning NHS Health Checks to local authorities in 2013. This has led to less involvement by general practice (Price, 2016).

For people aged over 75 there has been a health check programme since its introduction as part of the 1990 GP contract. However, the ways in which practices implemented this has been variable. We found that some practices offered a health check to all patients on their practice list who were aged over 75. Others only identified older persons who might be 'at risk' using a postal questionnaire, only approached patients who had not responded to a call for a routine health check or who had not 
visited their GP in the last year. Where unmet needs were identified, patients were referred to a wide variety of services, but the highest percentage of referrals was to a GP.

In 2004 the introduction of the national QOF as part of the revised GP contract introduced performance payments for some screening activities theoretically providing an incentive to screen patients. There have been a number of studies on the impact of QOF, but few have looked specifically at the identification of new cases of a disease. We identified two studies that suggested that the effect of QOF on uncovering previously undiagnosed conditions has been both modest and geographically variable. The analysis suggested that there remained a considerable amount of undiagnosed CHD and hypertension in 2007, three years after the introduction of QOF (McLean et al., 2007). However, Dixon et al. (2011) in their study of general practices in deprived areas, found that practices were screening opportunistically patients with family members with particular conditions, or patients who looked obese or overweight. The exact effect of QOF on this is difficult to pin down, as these practices stated that they were doing this before QOF was introduced.

Beyond these national programmes and initiatives, there is a wide range of screening activity within general practice. Most of the screening activity for Chlamydia occurs in contraception/ sexual health services and general practice where both practice nurses and GPs offer the chlamydia test. We also found papers describing how practices involved receptionists in giving out leaflets or discussing screening with patients (McNulty et al., 2008; Freeman et al., 2009). Screening for alcohol misuse has also become increasingly important with GPs reporting asking about alcohol consumption 'all of the time' or 'most of the time' - but not routinely in situations where patients do not ask them first. However, some screening and brief interventions for alcohol misuse are also undertaken by a wide range of practitioners including pharmacists - but again not routinely. GPs have described feeling awkward about enquiring about alcohol consumption GPs may be unaware of potentially problematic drinking behaviour in patients whose alcohol consumption is only slightly above recommended levels (Rapley et al., 2006). As a result, some GPs embed the question in a list of questions about lifestyle, which they say they 'ask everyone'.

Primary Health Care Research \& Development 2017; 18: 529-540
Other screening activities identified in the review included antenatal screening for haemoglobin disorders, screening for dementia, falls, osteoporosis, atrial fibrillation, domestic violence, depression, adult hearing, hepatitis $\mathrm{C}$, human immunodeficiency virus, oral cancer, syphilis and tuberculosis. These activities have been driven by personal interest, organisational contexts, perceived needs, QOF and other local public health initiatives. General practice also has a supportive role with respect to screening programmes that are carried out outside the practice, such as the breast screening and bowel cancer programmes which GPs support by verbally recommending screening, or sending out letters of invitation or information leaflets to increase participation. We found that QOF and NHS Health Checks were clearly associated with an increase in systematic approaches to, and organisation of, primary and secondary prevention advice and activity - especially related to smoking, diet and lifestyle with an increasing use of nurses and healthcare assistants being employed in practices.

\section{Primary prevention}

The King's Fund identified primary prevention as a key element in transforming healthcare systems (Naylor et al., 2015). Primary care is seen as an ideal location for primary prevention given that most patient contacts occur in this setting. We identified 326 papers that were classified as primary prevention. Of these, 50 related to child health, 49 to smoking, 22 to vaccination, and 40 to exercise and weight. In addition to these there were a small number of papers relating to falls, diabetes and mental health. Some of these categories overlapped, providing higher numbers of papers for some topics. There were also a substantial number of papers focussing on child health (just under 205 of the total primary prevention papers). This specifically reflects the way child health services are organised in the United Kingdom where GPs, practice nurses, health visitors and school nurses provide a wide range of child health services in conjunction with community health services.

Studies on child health spanned a wide range of topics including accident prevention, breastfeeding, weight management and exercise. Many of the papers examined changes in patient behaviour as a 
result of an intervention. Studies fell into three broad categories:

1. Interventions either within practices or by the primary healthcare team that were aimed at identifying children who might be at risk of health problems, and these activities often targeted particular groups of children.

2. Interventions that were aimed at intermediary organisations such as training for teachers.

3. Interventions or activities in schools or the community.

Again few studies explicitly discussed the delivery and organisation of public health services. Primary care practitioners support national programmes such as the childhood vaccination programme (often delivered through schools) as this tends to support increased uptake. Conversely, GPs were less likely to engage in lifestyle advice for children.

One of the few papers that addressed the context of a public health intervention policy discussed the variance in outcomes of a RCT of a community based breastfeeding support group (Hoddinott et al., 2010). Through ethnographic enquiry of the complex intervention, they found there was variation in the way that services were organised, which could be explained through a Hierarchy of Service Attributes Model. In the study sites where breastfeeding rates increased, the model was underpinned by good personnel resources and organisational stability, and demonstrated reflective action cycles at its apex. In localities where breastfeeding declined, the authors found that managers focussed on solving the problems within the model, such as staff shortages, rather than ways to deliver the policy. The model provides a useful approach to understanding and explaining delivery of public health interventions in primary care and could be usefully applied to other scenarios.

There were 49 papers on smoking cessation covering a wide range of interventions, and it was not always possible to distinguish between those interventions which targeted all smokers (primary prevention) and those that focussed on smokers who already had an existing health problem (secondary prevention). There is good evidence to suggest that services for helping people to quit smoking are clinically and cost effective. Almost all GPs (98\%) say that they follow NICE guidelines to ask patients about their smoking status and record this in patient records - although a number of studies highlighted poor recording of smoking status. Compliance with NICE guidelines was driven by the inclusion of payment criteria in QOF but despite this, fewer than half of GPs consistently advise patients to give up smoking. Although complex, the main reason seems to be concerned with damaging the relationship with the patient by raising the issue of smoking cessation, especially if the GP believes that the patient is not motivated enough to quit (Pilnick and Coleman, 2010). Overall, few papers examined the organisation and delivery of smoking cessation services, and only two compared different service approaches. Although there were many smoking cessation activities taking place in general practice, there was little evidence on how best to organise services.

There were 40 papers on exercise, weight reduction and diet which were mainly aimed at a general adult population, although one referred specifically to people with mental health issues and was therefore excluded, and there were three papers that focussed on older people. The majority of papers on weight loss were related to secondary prevention or identifying risk factors for a health issue - usually CVD. Interventions ranged from advice about lifestyle to specific recommendations concerning exercise or weight loss classes. However, there were very few good quality studies. In particular, those studies examining weight loss and obesity were generally of low methodological quality. Although the GP is a trusted source of advice regarding diet, they are rarely the first port of call for overweight and obese people. The evidence suggested that GPs regarded adult obesity as being the responsibility of the patient, and felt they had very limited ability to effect changes in their patients' diet or behaviour (Ogden and Flanagan, 2008). In addition, many GPs were hesitant to raise the issue of weight loss with overweight and obese patients if they feel it will negatively impact on their relationship with the patient (Hankey et al., 2003), and many believe that it does not belong within the medical domain. On the whole interventions and referrals tended to be undertaken haphazardly (by both GPs and practice nurses). Recent research on changes in the public health system in England identified a number of problems related to the organisation and commissioning of services for helping obese and overweight people - particularly since the reorganisation of public health services in 2013 (Peckham et al., 2015). 


\section{Secondary prevention}

It was not always easy to separate primary from secondary prevention as there was often insufficient information in the papers reviewed about whether patient groups were symptomatic or asymptomatic. Thus many aspects of secondary prevention were also covered in papers on primary prevention. Of those papers specifically classified by the research team as secondary prevention, most (43) addressed CVD (including health checks), and there were 25 papers addressing weight and exercise for people with symptomatic disease.

Studies that examined services for cardiovascular prevention mainly focussed on attempts to modify lifestyle risk factors in both symptomatic and asymptomatic patients but doctors did not always follow guidelines and procedures. Risk factors included obesity, poor diet, lack of exercise alcohol use and smoking. Other areas included monitoring of blood pressure in symptomatic patients - especially those with peripheral arterial disease, and patient compliance with medications, especially statins. Ten papers specifically addressed lifestyle risk factors in asymptomatic patients, of which five also looked at trying to change people's behaviour, such as encouraging exercise, or changing to a healthier diet or smoking cessation. They covered a range of interventions including Tai Chi, exercise classes, guided walks, counselling sessions, and in one case, a home exercise kit. Few papers were more than descriptive with no assessment of the best delivery or organisational methods for delivery or how services were selected. Few papers specifically looked at gender and CVD. These studies showed that women tended to receive suboptimal care with regard to prescribing and diagnostic tests, risk factor assessment, secondary prevention therapy, cardiac investigation and coronary revascularisation.

Two papers examined ethnicity and CVD. Murray et al. (2010) examined a 10 year strategy to reduce health inequalities in Wandsworth, London. However, there was insufficient data in the paper to assess whether this had actually made any impact on health inequalities. The second study examined the feasibility of using screening to reach hard to reach groups in Sandwell. Although the screening seemed popular, it was unclear if this had actually resulted in any lifestyle risk factor change (Patel et al., 2007). However, these papers like many of those reviewed did not provide sufficient detail, about how the intervention was provided (by who, where, when, etc.).

As stated earlier, most papers on preventive interventions did not provided sufficient details about the organisation and delivery of those interventions to assess their effectiveness. One exception was in relation to welfare advice given in general practice by trained staff from organisations such as the Citizens Advice Bureaux or by specialist welfare advisors. These papers concluded that it was an effective intervention and a way to address the social, economic and environmental influences on the health of their practice population. Such services tended to be highly valued by both patients and the practice staff and lead to positive outcomes for patients (Abbott et al., 2006; Burrows et al., 2011).

\section{Discussion}

Our review found that the range and type of activities undertaken in general practice was diverse. However, we found that little attention has been paid to examining the impact of the organisational context on the way services are delivered or how this affects the effectiveness of prevention and health promotion interventions in general practice. The focus of most studies was on individual approaches for medical conditions related to specific disease prevention such as diabetes or CHD with practices engaging in both primary and secondary prevention. Activity does appear to be driven by specific contractual incentives and conditions - such as the $\mathrm{QOF}$ - as well as national policies and programmes.

The evidence base is very limited and the studies we examined were mixed in terms of detail and methodological quality. There is insufficient good quality evidence to draw clear conclusions about many areas of prevention and health promotion practice in general practice. It was often impossible to identify sufficient information about the context and organisation of the delivery of prevention and health promotion prevention and health promotion interventions. Of particular concern was that few high quality studies were identified in key areas of primary prevention with the exception of smoking cessation services where services to support quitting were viewed as effective. A key message though is that multiple risk factor interventions in primary prevention comprising counselling, education and 
drug treatments were more likely to be effective in high-risk groups than in the general population. Although the provision of secondary prevention could be improved by using specific disease management programmes, the optimal mix of their components remains uncertain. There is insufficient evidence to make clear decisions about the balance between universal, opportunistic and targeted health promotion interventions. The cost-effectiveness of preventive interventions and the impact of how they are organised and delivered was difficult to determine due to the diverse nature of the interventions (type and organisation, different target groups) and research methodology and the resultant dearth of cost-effectiveness studies. Studies also tended to focus on specific interventions delivered by specific professionals (usually GPs or nurses) with virtually no comparative studies of different delivery or organisational models. Surprisingly few studies related to alternative models of delivery such as Community Oriented Primary Care (COPC). Though COPC has been trialled in many countries, it has yielded little evaluative research (Gillam and Schamroth, 2002; Gavagan, 2008).

Given the increasingly diverse nature of delivery within practices (through the use of practice nurses, healthcare assistants, counsellors and pharmacists) as well as some activities being shifted outside the practice (such as in schools, smoke-stop services and pharmacies), or delivered by non-practice staff (such as pharmacists, lay workers), studies that compare the relative effectiveness of different models are urgently needed. One exception was the research reported by Hoddinott et al. (2010) on the variance of change in relation to breastfeeding groups. Their Hierarchy of Services Attributes model provides an approach that can help explain why and how the organisation and delivery of an intervention is contextually significant and could be applied to other prevention and health promotion prevention and health promotion scenarios.

\section{Future priorities for research}

Currently, the major areas of research tend to be driven by clinical interest and we need more discussion about the type and range of disease burdens in primary care. We need to move away from areas such as CVD where rates are declining to examine areas with chronic disease burdens, for example cancer, dementia, other disabilities of old age. In particular, Although the QOF was associated with primary and secondary prevention activities and more systematic approaches to screening and prevention interventions it also influenced the focus of research.

We found substantial numbers of cross-sectional studies examining patient and professional views but less research that examined what was being done and how. Future research is needed that examines the whole prevention pathway for health problems that are managed within primary care drawing together research from general practice, pharmacy, community engagement, etc. This review did not cover mental health. Addressing mental health issues remains a major area of activity in general practice and a future review should address this area of prevention and health promotion activity. Studies are required that examine interventions for a population group (eg, older people) or an intervention type (eg, welfare advice) rather than just a specific clinical intervention. Research that more fully examines the impact of prevention and health promotion activities on health inequalities is also needed as this was seldom examined in the studies we reviewed and the focus on screening and on reactive interventions for secondary care may exacerbate health inequalities (Lorant et al., 2002; Capewell and Graham, 2010).

Research on prevention and health promotion in general practice also needs to move beyond clinical research to include delivery systems and be conducted in a primary care context to ensure that it is relevant and more likely to be transferred to practice. One important driver for primary care practitioner involvement in research is the need to improve quality of care. This could be achieved by the development of a programme of research underpinned by Improvement Science (Marshall et al., 2013). This would enable researchers and primary care practitioners to work together to implement and evaluate intervention and innovations to promote and improve health within its organisational context.

\section{Limitations}

This was a complex review to undertake due to problems about definition of terms and also because of the diversity of the topic. The research team made substantial efforts to identify appropriate search terms and develop inclusion and exclusion criteria but the volume of papers limited

Primary Health Care Research \& Development 2017; 18: 529-540 
the extent to which paper selection was cross checked between researchers. Although we made every effort to ensure consistency in searching and paper selection we recognise that our eventual strategy produced a particular set of results and that it is possible that alternative search strategies could have produced a different set of results in some areas of public health activity. Based on the experience of this review it is possible that undertaking a number of more narrowly focussed reviews may be beneficial but our results suggest that there would still be very little data on the delivery and organisation of prevention and health promotion activities. Our conclusions about the delivery and organisation of prevention and health promotion activities were also limited by the often scant information provided in the published papers. There were papers that examined different interventions by different practitioners (eg, screening by nurses or GPs, GPs or pharmacists undertaking health checks) and that discussed the provision of activities in different, specific locations such as the practices, schools or pharmacies but few papers made any comparison between these to allow a comprehensive analysis of the relative effectiveness of interventions.

\section{Conclusion}

There is currently insufficient evidence to support the most effective organisation and delivery of the prevention and health promotion interventions undertaken in general practice and primary care. This is not to conclude that there are no interventions that improve population health; for example, brief interventions for stopping smoking are effective. Some evidence supports specific interventions being undertaken with some patient groups and in some locations. In particular there is very little evidence on the most effective way to organise and deliver services and further research is needed to understand what the most effective organisational models of delivery are.

\section{Acknowledgements}

We acknowledge the help of Dr Kiran Nanchahal, Ben Ritchie, Dr Rebecca Rogers, Dr Andrew Wallace who helped with early stages of this study

Primary Health Care Research \& Development 2017; 18: 529-540 and Jane Falconer from LSHTM library who undertook the literature searches.

\section{Financial Support}

This project was funded by the National Institute for Health Research (Health Services and Delivery Programme) (project number 09/1001/52).

\section{Disclaimer}

The views and opinions expressed therein are those of the authors and do not necessarily reflect those of the (Health Services and Delivery Programme). NIHR, NHS or the Department of Health.

\section{Supplementary material}

To view supplementary material for this article, please visit https://doi.org/10.1017/S146342361700 0494

\section{References}

Abbott, S., Hobby, L. and Cotter, S. 2006: What is the impact on individual health of services in general practice settings which offer welfare benefits advice? Health Social Care Community 14, 1-8.

Anderson, S., Allen, P., Peckham, S. and Goodwin, N. 2008: Asking the right questions: scoping studies in the commissioning of research in the organisation and delivery of health services. Health Research Policy Systems 6, 7.

Arksey, H. and O'Malley, L. 2005: Scoping studies: towards a methodological framework. International Journal of Research Methods 8, 19-32.

Brady, T.J. 2013: A meta-analysis of health status, health behaviors, and health care utilization outcomes of the chronic disease self-management program. Preventing Chronic Disease 10, Published online 2013 Jan 17, E07. doi: 10.5888/pcd10.120112.

Brotons, C., Björkelund, C., Bulc, M., Ciurana, R., GodyckiCwirko, M., Jurgova, E., Kloppe, P., Lionis, C., Mierzecki, A., Piñeiro, R. and Pullerits, L. 2005: Prevention and health promotion in clinical practice: the views of general practitioners in Europe. Preventive Medicine 40, 595-601.

Burrows, J., Baxter, S., Baird, W., Hirst, J. and Goyder, E. 2011: Citizens advice in primary care: a qualitative study of the views and experiences of service users and staff. Public Health 125, 704-10.

Capewell, S. and Graham, H. 2010: Will cardiovascular disease prevention widen health inequalities? PLoS Medicine 7, e1000320.

Capewell, S., McCartney, M. and Holland, W. 2015: Invited debate: NHS Health Checks - a naked emperor? Journal of Public Health 37, 187-92. 
Dixon, A., Khachatryan, A., Wallace, A., Peckham, S., Boyce, T. and Gillam, S. 2011: The Quality and Outcomes Framework: does it reduce health inequalities? Final report. NIHR Service Delivery and Organisation Programme, Southampton.

Freeman, E., Howell-Jones, R., Oliver, I., Randall, S., Ford-Young, W., Beckwith, P. and McNulty, C. 2009: Promoting chlamydia screening with posters and leaflets in general practice: a qualitative study. BMC Public Health 9, 383.

Gavagan, T. 2008: A systematic review of COPC: evidence for effectiveness. Journal of Health Care for the Poor and Underserved 19, 963-80.

Gillam, S. and Schamroth, A. 2002: Community-oriented primary care experience in the United Kingdom. American Journal of Public Health 92, 1721-5.

Hankey, C.R., Eley, S., Leslie, W.S., Hunter, C.M. and Lean, M.E. 2003: Eating habits, beliefs, attitudes and knowledge among health professionals regarding the links between obesity, nutrition and health. Public Health Nutrition 7, 337-43.

Health Education England 2015: Primary Care Workforce Commission, London: HEE.

Health and Social Care Information Centre 2012: Cervical screening programme, England 2011-12. Leeds: Health and Social Care Information Centre.

HM Government 2010: The Coalition: our programme for government. London: Cabinet Office Retrieved April 1, 2014 from http://www.cabinetoffice.gov.uk/media/409088/ pfg_coalition.pdf.

Hoddinott, P., Britten, J. and Pill, R. 2010: Why do interventions work in some places and not others: a breastfeeding support group trial. Social Science and Medicine 70, 769-8.

Jackson, N. and Waters, E. 2004: Guidelines for systematic reviews of health promotion and public health interventions taskforce. The challenges of systematically reviewing public health interventions. Journal of Public Health 26, 303-7.

Langdown, C. and Peckham, S. 2013: The use of financial incentives to help improve health outcomes: is the quality and outcomes framework fit for purpose? A systematic review. Journal of Public Health 36, 251-8.

Lorant, V., Boland, B., Humblet, P. and Deliège, D. 2002: Equity in prevention and health care. Journal of Epidemiology and Community Health 56, 510-6.

Marshall, M., Pronovost, P. and Dixon-Woods, M. 2013: Promotion of improvement as a science. Lancet 381, 419-21.

McLean, G., Guthrie, B. and Sutton, M. 2007: Differences in the quality of primary medical care for CVD and diabetes across the NHS: evidence from the Quality and Outcomes Framework. BMC Health Services Research 7, 74.

McNulty, C.A., Freeman, E., Oliver, I., Ford-Young, W. and Randall, S. 2008: Strategies used to increase chlamydia screening in general practice: a qualitative study. Public Health 122, 845-56.

Murray, J., Saxena, S., Millett, C., Curcin, V., de Lusignan, S. and Majeed, A. 2010: Reductions in risk factors for secondary prevention of coronary heart disease by ethnic group in south-west London: 10-year longitudinal study (1998-2007). Family Practice 27, 430-8.

National Institute for Health and Clinical Excellence 2013: Physical activity: brief advice for adults in primary care. NICE Public Health Guidance no. 44 London: NICE.

NHS England 2014: Call to Action: Primary Care.

NHS England 2016: GP Forward View. London: NHSE.

Naylor, C., Imison, C. and Addicott, R. 2015: Transforming. London: King's Fund.

Ogden, J. and Flanagan, Z. 2008: Beliefs about the causes and solutions to obesity: a comparison of GPs to lay people. Patient Education Counselling 71, 72-8.

Patel, J.V., Gunarathne, A., Lane, D., Lim, H.S., Tracey, I., Panja, N.C., Lip, G.Y. and Hughes, E.A. 2007: Widening access to cardiovascular healthcare: community screening among ethnic minorities in inner-city Britain - the Healthy Hearts Project. BMC Health Services Research 7, 192.

Peckham, S., Falconer, J., Gillam, S., Hann, A., Kendall, S., Nanchahal, K., Ritchie, B., Rogers, R. and Wallace, A. 2015: The organisation and delivery of prevention and health promotion in general practice and primary care: a scoping study. Health Services and Delivery Research 3, 29.

Peckham, S. and Gousia, K. 2014: GP payment schemes review. London: PRUComm. www.prucomm.ac.uk.

Peckham, S., Hann, A. and Boyce, T. 2011: Health promotion and ill-health prevention: the role of general practice. Quality in Primary Care 19, 317-23.

Peckham, S. and Wallace, A. 2010: Pay for performance schemes in primary care: what have we learnt? Quality in Primary Care 18, 111-6.

Pilnick, A. and Coleman, T. 2010: Do your best for me: the difficulties of finding a clinically effective endpoint in smoking cessation consultations in primary care. Health (London) 14, 57-74.

Price, C. 2016: Local council strips GP practices of NHS Health Checks. Pulse, 29 January 2016. Retrieved 24 April 2016 from http://www.pulsetoday.co.uk/news/commissioning/local-councilstrips-gp-practices-of-nhs-health-checks/20031011.article.

Rapley, T., May, C. and Kaner, E.F. 2006: Still a difficult business? Negotiating alcohol-related problems in general practice consultations. Social Science \& Medicine 63, 2418-8.

Robertson, J., Roberts, H., Emerson, E., Turner, S. and Greig, R. 2011: The impact of health checks for people with intellectual disabilities: a systematic review of evidence. Journal of Intellectual Disability Research 55, 1009-9.

Royal College of General Practitioners (RCGP) 2007: Healthy People: promoting health and preventing disease. Retrieved 10 June 2010 from http://www.rcgp-curriculum.org.uk/pdf/ curr_5_Healthy_people.pdf.

Royal College of Nursing 2007: Nurses as partners in delivering public health. London: Royal College of Nursing.

Rubio-Valera, M., Pons-Vigués, M., Martínez-Andrés, M., Moreno-Peral, P., Berenguera, A. and Fernández, A. 2014: Barriers and facilitators for the implementation of primary prevention and health promotion activities in primary care: a synthesis through metaethnography. PLoS One 9, e89554. 
Simmons, R.K., Echouffo-Tcheugui, J.B., Sharp, S.J., Sargeant, L.A., Williams, K.M., Prevost, A.T., Kinmonth, A.L., Wareham, N.J. and Griffin, S.J. 2012: Screening for type 2 diabetes and population mortality over 10 years (ADDITION-Cambridge): a cluster-randomised controlled trial. Lancet 380, 1741-8.

Waterall, J., Greaves, F., Kearney, M. and Fenton, K.A. 2015: Invited debate: NHS Health Check: an innovative component of local adult health improvement and well-being programmes in England. Journal of Public Health 37, 177-84.

Watson, M. 2008: Going for gold: the health promoting general practice. Quality in Primary Care 16, 177-85.

World Health Organization 1978: Declaration of Alma Ata. International conference on primary care; 6-12 September 1978. Alma Ata: USSR.

World Health Organization 2005: Primary care: now more than ever. Geneva: WHO. 